\title{
Formation Shape Control: Global Asymptotic Stability of a Four-Agent Formation
}

\author{
Tyler H. Summers ${ }^{\dagger}$, Changbin Yu ${ }^{\ddagger}$, Brian D.O. Anderson ${ }^{\ddagger}$, and Soura Dasgupta ${ }^{\S}$ \\ ${ }^{\dagger}$ University of Texas at Austin $\quad{ }^{\ddagger}$ The Australian National University ${ }^{\S}$ University of Iowa
}

\begin{abstract}
This paper considers formation shape control of a team of four agents in the plane, motivated by an example from [1]. We utilize bidirectional, gradient-based interagent distance control laws which are designed so that the agents cooperatively achieve a specified desired formation shape. When every interagent distance is actively controlled (i.e. the information architecture is a complete graph), there may exist equilibrium formation shapes with incorrect interagent distances. We prove that any incorrect equilibrium formation shape is locally unstable for formations with internal angles that satisfy an acuteness condition and thereby establish that the desired formation shape, for all practical purposes, is globally asymptotically stable.
\end{abstract}

\section{INTRODUCTION}

Autonomous vehicle formations and mobile sensor networks have ubiquitous potential for scientific and engineering applications and present unique technical challenges. Applications include teams of UAVs performing military reconnaissance and surveillance missions in hostile environments, satellite formations for high-resolution Earth and deepspace imaging, and submarine swarms for oceanic exploration and mapping. A fundamental task for autonomous vehicle formations is formation shape control. Precisely controlled formations can maintain mobile agents in optimal sensing configurations.

In this paper, the formation shape is controlled by actively controlling a certain set of interagent distances using relative position measurements. The objective is to design motion control laws for each agent such that the agents cooperatively and autonomously achieve a specified desired formation shape. Early work within this framework includes [2]-[5]. In [2], Eren et al propose the use of graph rigidity theory (see e.g. [6]) for modeling information architectures. In [3] and [4], Olfati-Saber and Murray also utilize graph rigidity theory and propose gradient control laws based on structural potential functions which are generated from the graph. In [5], Baillieul and Suri also utilize graph rigidity theory and discuss application to formations of non-holonomic robots.

T. H. Summers is a Fulbright Postgraduate Scholar supported by the Australian-American Fulbright Commission. C. Yu is supported by the Australian Research Council through an Australian Postdoctoral Fellowship under DP-0877562. B.D.O. Anderson is supported by DP-0877562 and by National ICT Australia-NICTA. NICTA is funded by the Australian Government as represented by the Department of Broadband, Communications and the Digital Economy and the Australian Research Council through the ICT Centre of Excellence program. S. Dasgupta is with the Department of Electrical and Computer Engineering, University of Iowa, Iowa City, IA 52242, USA, He was a visitor with NICTA and was also supported by NSF grants ECS-0622017, CCF-07290 and CCF-0830747. Email: thsummers@mail.utexas.edu,\{brad.yu,brian.anderson $\} @$ anu.edu.au, dasgupta@engineering.uiowa.edu
More recently, in [1] Krick et al provide a complete analysis showing that the desired formation shape is locally asymptotically stable under a gradient control law, provided that the information architecture is rigid. They emphasize that the desired formation shape is a three-dimensional equilibrium manifold, and therefore since the resulting linearized system is non-hyperbolic, a non-trivial application of center manifold theory is required. The global stability properties of the desired formation shape remain a challenging open problem. An interesting simulation from [1] that touches on global stability properties involves a four-agent formation with a complete graph information architecture (i.e. all interagent distances are actively controlled). The formation appears to converge to an incorrect shape (whereby incorrect shape refers to a formation with interagent distances not all the same as those in the desired shape), and they conclude that the desired shape is not globally asymptotically stable.

In this paper, we elaborate on this four-agent example from [1]. In particular, we show that the observed incorrect equilibrium formation is in fact an unstable saddle. More generally, we show that under certain acuteness conditions on the desired formation shape, any possible incorrect equilibrium shape is unstable and thereby establish that the desired shape is almost globally asymptotically stable. That is, although there exist some trajectories which may converge to the incorrect shape, the initial conditions that give rise to such trajectories belong to a set of measure zero; any infinitesimally small perturbation will cause divergence from these trajectories, and the agents will converge to the desired shape.

The paper is organized as follows. Section II presents the equations of motion for the four-agent formation shape control problem and examines the example from [1]. We provide an easily checkable condition for local instability of an equilibrium shape and consider the general class of rectangular desired formations. In Section III, we consider the most general classes of four-agent formations and prove our main result. Section IV gives some concluding remarks and future research considerations.

\section{EQUATIONS OF MOTION AND EXAMPLES}

In this section, we present equations of motion for the fouragent formation shape control problem. We then examine the example from [1] that illustrates existence of an incorrect equilibrium formation shape and show that this shape is an unstable saddle. 

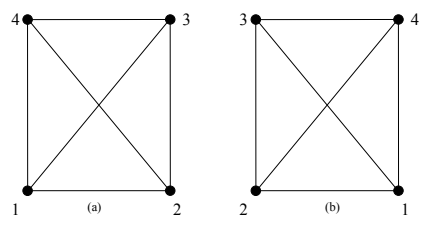

Fig. 1. A desired formation configuration and its reflection in the plane both satisfy the same prescribed interagent distance constraints.

\section{A. Equations of Motion}

Let $p=\left[p_{1}, p_{2}, p_{3}, p_{4}\right]^{T} \in \Re^{8}$ be a vector of the four agent positions in the plane. Following [1], we use a single integrator agent model to describe the motion of each agent $\dot{p}_{i}=u_{i}$ where $u_{i}$ is the control input to be specified. Let $d=$ $\left[d_{12}, d_{13}, d_{14}, d_{23}, d_{24}, d_{34}\right]^{T}$ be a vector of desired interagent distances that define the formation shape and are to be actively controlled. We assume that the entries of $d$ correspond to a realizable shape.

We define the rigidity function as a vector of the current interagent distances corresponding to the entries of $d$

$$
\begin{array}{r}
r(p)=\left[\left\|p_{1}-p_{2}\right\|^{2},\left\|p_{1}-p_{3}\right\|^{2},\left\|p_{1}-p_{4}\right\|^{2},\right. \\
\left.\quad\left\|p_{2}-p_{3}\right\|^{2},\left\|p_{2}-p_{4}\right\|^{2},\left\|p_{3}-p_{4}\right\|^{2}\right]^{T}
\end{array}
$$

and the error function

$$
e(p)=r(p)-d^{2}=\left[e_{12}, e_{13}, e_{14}, e_{23}, e_{24}, e_{34}\right]^{T}
$$

where the entries of $d^{2}$ are the squares of the entries of $d$. The desired formation shape is a three-dimensional manifold in $\Re^{8}$ given by

$$
P_{d}=\left\{p \in \Re^{8} \mid p=r^{-1}\left(d^{2}\right)\right\}
$$

which is non-empty for a realizable $d$. Note the following symmetry for any planar formation: two distinct formation orientations both correspond to a correct formation shape: one orientation and its reflection in the plane, as illustrated in Figure 1.

Now consider the potential function

$V(p)=\frac{1}{2}\|e(p)\|^{2}=\frac{1}{2}\left(e_{12}^{2}+e_{13}^{2}+e_{14}^{2}+e_{23}^{2}+e_{24}^{2}+e_{34}^{2}\right)$.

The function quantifies the total interagent distance error between the current formation and the desired formation $d$. Note that $V \geq 0$ and $V=0$ if and only if $e(p)=0$, that is if and only if the formation is in the desired shape. Thus, $V$ is a suitable potential function from which to derive a gradient control law. Accordingly, let the control input be given by

$$
u=-\nabla V(p)^{T} .
$$

Then the closed-loop system is given by

$$
\dot{p}=-\nabla V(p)^{T}=-\left[J_{r}(p)\right]^{T} e(p)
$$

where $J_{r}(p)$ is the Jacobian of the rigidity function $r(p)$ (also known as the rigidity matrix). This can be expressed in the following form

$$
\dot{p}=-\left(E(p) \otimes I_{2}\right) p
$$

where the matrix $E(p)$ is given by

$$
\begin{aligned}
& E(p)= \\
& {\left[\begin{array}{cccc}
e_{12}+e_{13}+e_{14} & -e_{12} & -e_{13} & -e_{14} \\
-e_{12} & e_{12}+e_{23}+e_{24} & -e_{23} & -e_{24} \\
-e_{13} & -e_{23} & e_{13}+e_{23}+e_{34} & -e_{34} \\
-e_{14} & -e_{24} & -e_{34} & e_{14}+e_{24}+e_{34}
\end{array}\right]}
\end{aligned}
$$

where $\otimes$ is the Kronecker product. The equilibrium points of the closed-loop system (4) are the same as the critical points of the potential function $V$. The Jacobian of the right side of (4), which we denote as $J_{f}(p)$, is the same as the negative of the Hessian of $V$, which we denote as $H_{V}(p)$. These are given by

$$
H_{V}(p)=2 J_{r}(p)^{T} J_{r}(p)+E(p) \otimes I_{2}=-J_{f}(p) .
$$

Therefore, a study of the stability of the equilibrium points of (4) amounts to a study of the nature of the critical points of $V$ : minima are locally stable and maxima and saddle points are locally unstable. Note that the stability of an equilibrium point is independent of rotation and translation of the formation shape. In particular, only relative positions matter.

\section{B. Krick Example}

In [1], Krick et al prove for an $n$-agent formation that under the gradient control law (3), the desired formation is locally asymptotically stable (in the sense that for $\|e(p(0))\|$ sufficiently small, $\|e(p(t))\| \rightarrow 0$ as $t \rightarrow \infty)$ if the underlying information architecture is rigid. Since the desired formation shape given by (2) is a three-dimensional equilibrium manifold, the linearized system is non-hyperbolic and they utilize center manifold theory to obtain the result.

The global stability properties remain a challenging open problem, and in exploring this issue Krick et al give an interesting four-agent example with $K_{4}$ information architecture where the formation appears to converge to an incorrect equilibrium formation shape. The example is as follows. Suppose the desired formation is a $1 \times 2$ rectangle given by $d^{2}=[1,5,4,4,5,1]^{T}$. It is easy to verify that when we use the desired distances specified by $d$, any formation with distance set $d_{1}^{2}=\left[\frac{11}{3}, \frac{7}{3}, \frac{4}{3}, \frac{4}{3}, \frac{7}{3}, \frac{11}{3}\right]^{T}$ is also an equilibrium with incorrect interagent distances. The incorrect equilibrium is a "twisted rectangle" with the term twisted referring to a change in agent ordering, as illustrated in the middle formation of Figure 2 (it turns out there is a further incorrect equilibrium shape, which we discuss later). Krick et al concluded that the desired shape is not globally attractive since the control law appears to cause convergence to an incorrect equilibrium shape. However, when we evaluate the eigenvalues of the negative of the Hessian evaluated at the incorrect shape (i.e. eig $\left[J_{f}\left(p^{*}\right)\right]=$ eig $\left[-H_{V}\left(p^{*}\right)\right]$ where $p^{*}$ satisfies $\left.p^{*}=r_{-1}\left(d_{1}^{2}\right)\right)$, we obtain $\{0,0,0,-22.78,-14.67,-6.56,1.33,5.33\}$. Since there are both positive and negative eigenvalues, then the incorrect equilibrium shape is in fact a saddle and is therefore unstable. 


\section{General Rectangular Formations}

We can generalize the preceding example to the case where the desired formation is a rectangle of arbitrary size. There are two possible incorrect equilibrium shapes which manifest as "twisted rectangles" with a change in agent ordering.

Let us first present a sufficient condition for local instability of an equilibrium formation shape that can be checked more easily than the eigenvalue condition. The result, applicable without any restriction to a rectangular desired shape, is as follows.

Lemma 1. Let $p^{*}$ be an equilibrium point of the closed-loop system (4). Suppose the sum of errors at $p^{*}$ is negative, that is $e_{\text {sum }}\left(p^{*}\right)=e_{12}+e_{13}+e_{14}+e_{23}+e_{24}+e_{34}<0$. Then $p^{*}$ is an unstable equilibrium of (4).

Proof: Because of the form of the closed-loop system (4), an equilibrium $p^{*}$ is unstable if the matrix $-E\left(p^{*}\right)$ has eigenvalues in the right half-plane. (*LATE NOTE: We have recently realized that there is a logical gap in this claim that requires some rethinking and further investigation in order to guarantee the following results. This is currently underway.) From (5), the trace of this matrix is given by

$$
\operatorname{tr}\left(-E\left(p^{*}\right)\right)=-2 e_{\text {sum }}\left(p^{*}\right)
$$

If $e_{\text {sum }}\left(p^{*}\right)<0$, then $\operatorname{tr}\left(-E\left(p^{*}\right)\right)>0$, and since the trace of a matrix is the sum of its eigenvalues, then at least one eigenvalue of $-E\left(p^{*}\right)$ must be in the right half-plane.

Now suppose that the desired formation is an $a \times b$ rectangle given by $d=\left[a^{2}, a^{2}+b^{2}, b^{2}, b^{2}, a^{2}+b^{2}, a^{2}\right]^{T}$. It is easy to verify that the "twisted" rectangle given by

$$
d_{1}=\left[a^{2}+\frac{2}{3} b^{2}, a^{2}+\frac{1}{3} b^{2}, \frac{1}{3} b^{2}, \frac{1}{3} b^{2}, a^{2}+\frac{1}{3} b^{2}, a^{2}+\frac{2}{3} b^{2}\right]^{T}
$$

is an incorrect equilibrium shape. Similarly, the "twisted" rectangle given by

$$
d_{2}=\left[\frac{1}{3} a^{2}, \frac{1}{3} a^{2}+b^{2}, \frac{2}{3} a^{2}+b^{2}, \frac{2}{3} a^{2}+b^{2}, \frac{1}{3} a^{2}+b^{2}, \frac{1}{3} a^{2}\right]^{T}
$$

is also an incorrect equilibrium shape. The desired and both twisted equilibrium shapes are illustrated in Figure 2 for the Krick example where $a=1$ and $b=2$.

A simple calculation with (1) gives the interagent distance errors for each of the incorrect equilibrium shapes. The sum of errors for the incorrect equilibrium shape $d_{1}$ is $\sum_{i} \sum_{j} e_{i j}=-\frac{2}{3} b^{2}<0$, and the sum of errors for the incorrect equilibrium shape $d_{2}$ is $\sum_{i} \sum_{j} e_{i j}=-\frac{2}{3} a^{2}<0$. Thus, by Lemma 1, both are unstable.

If one could show that any such incorrect equilibrium shape is unstable, then the desired formation shape would be almost globally asymptotically stable. That is, all initial conditions, apart from a thin set that may converge to a saddle equilibrium, would converge to the desired shape. We will show in the rest of this paper that this is indeed the case for any four-agent formation under certain acuteness conditions on the internal angles of the desired formation.

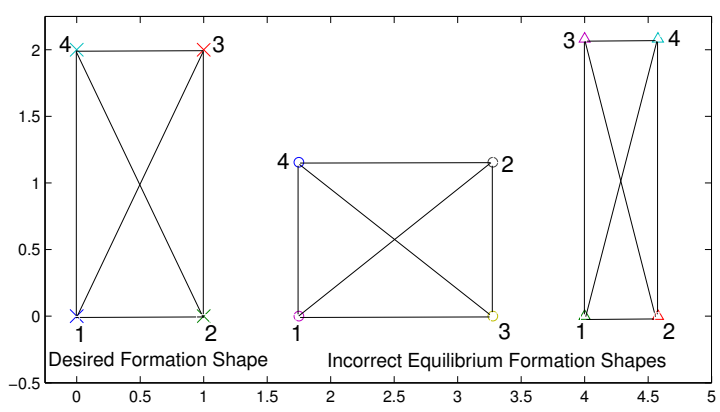

Fig. 2. The desired formation is rectangular and there are two possible "twisted rectangles" that are incorrect equilibrium shapes. Note that each formation has two different pairs of agents on the diagonals of the rectangle: (a) 13 and 24, (b) 12 and 34, and (c) 14 and 23.

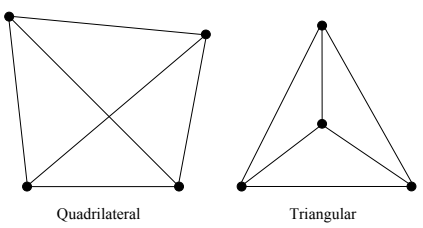

Fig. 3. In general, four-agent formations can be (a) quadrilateral or (b) triangular.

\section{General Four-Agent Formations}

In this section, we consider general four-agent formations. As illustrated in Figure 3, formations of four agents can assume two non-degenerate types: quadrilateral, where the four agents are corners of a convex set, and triangular, where one agent is inside the triangular convex hull of the three other agents. Degenerate formations have a collinearity of three or four agents or a collocation of two or more agents. We will assume that the desired formation is non-degenerate.

We first present two results that give conditions for a quadrilateral or triangular incorrect equilibrium shape to be stable. These conditions are independent of the desired formation shape. We then present cases for the various possible combinations of desired and incorrect formation shapes and show that under certain acuteness assumptions on the desired formation, the conditions for incorrect equilibrium shapes to be stable result in contradictions. We give similar results for degenerate incorrect formation shapes. It follows that under the acuteness assumptions on the desired shape, all incorrect shapes are in fact unstable, and thus the desired shape is almost globally asymptotically stable.

Throughout the following analysis, we denote the interagent distances in the desired formation and in an incorrect equilibrium shape as $d_{i j}$ and $D_{i j}$, respectively.

Lemma 2. Suppose the quadrilateral in Figure 4(b) represents a stable incorrect equilibrium shape. Then there must hold

$$
\begin{gathered}
D_{12}<d_{12}, D_{34}<d_{34}, \\
D_{13}>d_{13}, D_{14}>d_{14}, D_{23}>d_{23}, D_{24}>d_{24} .
\end{gathered}
$$


Proof: Consider Figure 4(b). From equilibrium at agent $1^{\prime}$, we have

$$
e_{12}\left(p_{2^{\prime}}-p_{1^{\prime}}\right)+e_{13}\left(p_{3^{\prime}}-p^{\prime} 1\right)+e_{14}\left(p_{4^{\prime}}-p_{1^{\prime}}\right)=0 .
$$

A point on the line between agents $3^{\prime}$ and $4^{\prime}$, call it $p_{\lambda}$, satisfies

$$
p_{\lambda}=\lambda p_{3^{\prime}}+(1-\lambda) p_{4^{\prime}}, \quad \lambda \in(0,1) .
$$

We have

$$
\begin{aligned}
p_{\lambda}-p_{1^{\prime}} & =\lambda\left(p_{3^{\prime}}-p_{1^{\prime}}\right)+(1-\lambda)\left(p_{4^{\prime}}-p_{1^{\prime}}\right) \\
p_{2^{\prime}}-p_{1^{\prime}} & =\alpha\left(p_{\lambda}-p_{1^{\prime}}\right)
\end{aligned}
$$

for some $\alpha>1$ and some $\lambda \in(0,1)$. Combining (9) with (8), we have

$\left(\alpha \lambda e_{12}+e_{13}\right)\left(p_{3^{\prime}}-p_{1^{\prime}}\right)+\left(\alpha(1-\lambda) e_{12}+e_{14}\right)\left(p_{4^{\prime}}-p_{1^{\prime}}\right)=0$ and so

$$
\begin{aligned}
\alpha \lambda e_{12}+e_{13} & =0 \\
\alpha(1-\lambda) e_{12}+e_{14} & =0 .
\end{aligned}
$$

Adding, we have

$$
\alpha e_{12}+e_{13}+e_{14}=0
$$

which may be expressed as

$$
e_{12}+e_{13}+e_{14}=(1-\alpha) e_{12} .
$$

Since $\alpha>1$,

$$
\operatorname{sgn}\left(e_{12}+e_{13}+e_{14}\right)=-\operatorname{sgn}\left(e_{12}\right) .
$$

By (10),

$$
\operatorname{sgn}\left(e_{12}\right)=-\operatorname{sgn}\left(e_{13}\right)=-\operatorname{sgn}\left(e_{14}\right) .
$$

A similar argument from the equilibrium condition at agent 2 yields

$$
\begin{aligned}
\operatorname{sgn}\left(e_{12}\right) & =-\operatorname{sgn}\left(e_{12}+e_{23}+e_{24}\right) \\
& =-\operatorname{sgn}\left(e_{23}\right)=-\operatorname{sgn}\left(e_{24}\right)
\end{aligned}
$$

and similar arguments from the equilibrium conditions at agents 3 and 4 yield

$$
\begin{aligned}
\operatorname{sgn}\left(e_{34}\right) & =-\operatorname{sgn}\left(e_{13}+e_{23}+e_{34}\right) \\
& =-\operatorname{sgn}\left(e_{14}+e_{24}+e_{34}\right) \\
& =\operatorname{sgn}\left(e_{12}\right)
\end{aligned}
$$

Combining (12), (13), (14), (15), and (16), we have

$$
\operatorname{sgn}\left(e_{\text {sum }}\right)=-\operatorname{sgn}\left(e_{12}\right)
$$

which implies via Lemma 1 that if $e_{12}>0$ at the incorrect equilibrium, then it is unstable. Note that if $e_{12}=0$, then by the above sign pattern all other errors are also zero, which implies that the equilibrium is correct. Thus, for the incorrect equilibrium to be stable, we must have $e_{12}<0$, and the sign pattern above gives the result.

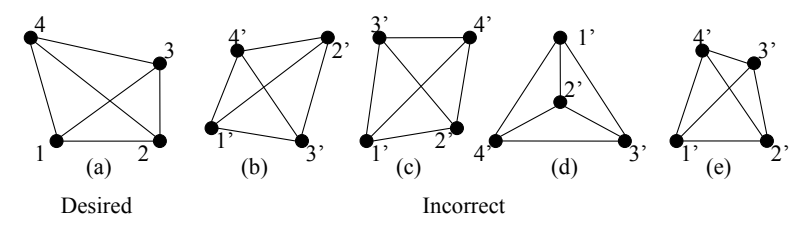

Fig. 4. The desired formation is the quadrilateral on the left and the other formations represent possible configurations for incorrect equilibrium shapes.

Lemma 3. Suppose the triangular formation in Figure 4(d) represents a stable incorrect equilibrium shape. Then there must hold

$$
\begin{aligned}
& D_{12}>d_{12}, D_{23}>d_{23}, D_{24}>d_{24}, \\
& D_{13}<d_{13}, D_{14}<d_{14}, D_{34}<d_{34} .
\end{aligned}
$$

Proof: The proof is nearly identical to that of Lemma 2 where now $\alpha<1$ and as a result we obtain the following sign pattern for the triangular formation

$$
\begin{aligned}
\operatorname{sgn}\left(e_{12}\right) & =\operatorname{sgn}\left(e_{23}\right)=\operatorname{sgn}\left(e_{24}\right)=\operatorname{sgn}\left(e_{\text {sum }}\right) \\
& =-\operatorname{sgn}\left(e_{13}\right)=-\operatorname{sgn}\left(e_{14}\right)=-\operatorname{sgn}\left(e_{34}\right) .
\end{aligned}
$$

For the triangular formation to be a stable equilibrium, we require $e_{12}>0$, and the above sign pattern gives the result.

\section{A. Quadrilateral Desired Formation}

We begin with the following lemma.

Lemma 4. Suppose the quadrilateral in Figure 4(a) represents the desired formation and the twisted quadrilaterals in Figure $4(b)$ and (c) and the triangular formation in Figure $4(d)$ represent incorrect equilibrium shapes. Suppose the diagonals in the desired formation make acute angles with the sides. Then the incorrect equilibrium shapes are all unstable.

Proof: Figure 4(b). Suppose the incorrect equilibrium shape in Figure 4(b) is stable. Then from Lemma 2, (7) holds. Now we claim that the angle $1^{\prime} 4^{\prime} 2^{\prime}$ must be acute. To establish a contradiction, suppose this angle is not acute. Then since angle 142 is acute by assumption, there holds

$$
\begin{aligned}
D_{12}^{2}-d_{12}^{2}= & D_{14}^{2}-d_{14}^{2}+D_{24}^{2}-d_{24}^{2}-2 D_{12} D_{24} \cos \left(1^{\prime} 4^{\prime} 2^{\prime}\right) \\
& +d_{12} d_{24} \cos (142)>0
\end{aligned}
$$

which contradicts $D_{12}<d_{12}$. Similarly, angles $1^{\prime} 3^{\prime} 2^{\prime}, 3^{\prime} 2^{\prime} 4^{\prime}$, and $4^{\prime} 1^{\prime} 3^{\prime}$ are acute and since the sum of these with $1^{\prime} 4^{\prime} 2^{\prime}$ is $2 \pi$, we have a contradiction. Thus, the incorrect equilibrium shape is unstable.

Figure 4(c). Same as above with agents 2 and 4 exchanging roles.

Figure $4(d)$. Suppose the incorrect equilibrium shape in Figure 4(d) is stable. Then from Lemma 3, (17) holds. Arguing again as before, angles $1^{\prime} 2^{\prime} 4^{\prime}$ and $3^{\prime} 2^{\prime} 4^{\prime}$ are both acute. Since their sum with $1^{\prime} 2^{\prime} 3^{\prime}$ is $2 \pi$, then $1^{\prime} 2^{\prime} 3^{\prime}>\pi$, which is impossible.

Remark. Proof of instability for incorrect triangular formations with either agent $1^{\prime}, 3^{\prime}$, or $4^{\prime}$ in the convex hull of the 

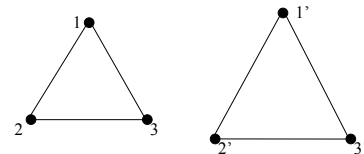

Fig. 5. Two triangles with different distance sets.

remaining three is almost identical to the last case above and is omitted.

We now consider the case in Figure 4(e) where an incorrect equilibrium shape has the same configuration as the desired shape (i.e. it is not twisted). We will make use of the following lemma.

Lemma 5. Consider the two triangles in Figure 5 and let $d_{i j}$ be interagent distances on the left and $D_{i j}$ be the interagent distances on the right. Suppose angles 123 and 132 are acute, $d_{12}<D_{12}, d_{13}<D_{13}$, and $d_{23}>D_{23}$. Then $2^{\prime} 1^{\prime} 3^{\prime}<213$.

Proof: We have

$D_{23}^{2}-d_{23}^{2}=D_{12}^{2}-d_{12}^{2}+D_{13}^{2}-d_{13}^{2}$ $-2 D_{12} D_{13} \cos \left(2^{\prime} 1^{\prime} 3^{\prime}\right)+2 d_{12} d_{13} \cos (213)<0$

Using the length inequalities, it follows that

$$
-2 D_{12} D_{13} \cos \left(2^{\prime} 1^{\prime} 3^{\prime}\right)+2 d_{12} d_{13} \cos (213)<0 .
$$

or equivalently

$$
\cos \left(2^{\prime} 1^{\prime} 3^{\prime}\right)>\frac{d_{12} d_{13}}{D_{12} D_{13}} \cos (213) .
$$

If $2^{\prime} 1^{\prime} 3^{\prime}$ is a right angle or is obtuse, then evidently $2^{\prime} 1^{\prime} 3^{\prime}<$ 213. If it is acute, we need a longer argument to show the same conclusion. First, it is immediate from this equation that $2^{\prime} 1^{\prime} 3^{\prime}$ is also acute. Further, since

$$
\frac{\sin (213)}{\sin (123)}=\frac{d_{23}}{d_{13}}>\frac{D_{23}}{D_{13}}=\frac{\sin \left(2^{\prime} 1^{\prime} 3^{\prime}\right)}{\sin \left(1^{\prime} 2^{\prime} 3^{\prime}\right)}
$$

whence

$$
\frac{\sin (213)}{\sin \left(2^{\prime} 1^{\prime} 3^{\prime}\right)}>\frac{\sin (123)}{\sin \left(1^{\prime} 2^{\prime} 3^{\prime}\right)}
$$

To obtain a contradiction, suppose that $2^{\prime} 1^{\prime} 3^{\prime} \geq 213$. Then $\sin \left(1^{\prime} 2^{\prime} 3^{\prime}\right)>\sin (123)$ Since 123 is acute, this means that $1^{\prime} 2^{\prime} 3^{\prime}>123$, whether or not $1^{\prime} 2^{\prime} 3^{\prime}$ is acute. A similar argument will show that $1^{\prime} 3^{\prime} 2^{\prime}>132$, and then the sum of the three angles of the triangle $1^{\prime} 2^{\prime} 3^{\prime}$ will exceed the sum for the triangle 123 , which is impossible. So $2^{\prime} 1^{\prime} 3^{\prime}<213$.

Now we have the following result.

Lemma 6. Suppose the quadrilateral in Figure 4(a) represents the desired formation and the quadrilateral in Figure 4(e) represents an incorrect equilibrium shape. Suppose the diagonals in the desired formation make acute angles with the sides. Then the incorrect equilibrium shape is unstable.

Proof: Suppose the incorrect equilibrium shape in Figure 4(e) is stable. With the new agent ordering, Lemma 2 now

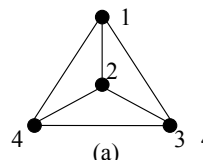

Desired

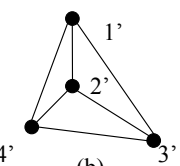

(b)

Incorrect

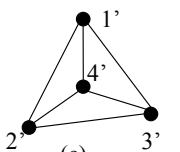

(c)

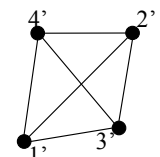

(d)
Fig. 6. The desired formation is the triangle on the left and the other figures represent possible configurations for incorrect equilibrium shapes.

yields

$$
\begin{gathered}
D_{13}<d_{13}, D_{24}<d_{24} \\
D_{12}>d_{12}, D_{14}>d_{14}, D_{23}>d_{23}, D_{34}>d_{34} .
\end{gathered}
$$

Then a direct application of Lemma 5 shows that angles $1^{\prime} 2^{\prime} 3^{\prime}, 2^{\prime} 3^{\prime} 4^{\prime}, 3^{\prime} 4^{\prime} 1^{\prime}$, and $4^{\prime} 1^{\prime} 2^{\prime}$ are smaller than angles 123 , 234,341 , and 412 , respectively, establishing a contradiction.

\section{B. Triangular Desired Formation}

Now we consider cases where the desired formation is triangular.

Lemma 7. Suppose the triangle in Figure 6(a) represents the desired formation and the triangles in Figure $6(b)$ and (c) represents an incorrect equilibrium shape. Suppose the angles 134, 341, and 413 are all acute. Then the incorrect equilibrium shapes are unstable.

Proof: Figure 6(b). Suppose the incorrect equilibrium shape in Figure 6(b) is stable. Then from Lemma 3, (17) holds. Then a direct application of Lemma 5 shows that angles 124,123 , and 324 are greater than angles $1^{\prime} 2^{\prime} 4^{\prime}, 1^{\prime} 2^{\prime} 3^{\prime}$, and $3^{\prime} 2^{\prime} 4^{\prime}$, respectively, leading to a contradiction.

Figure 6(c). Suppose the incorrect equilibrium shape in Figure 6(c) is stable. With the new agent ordering, Lemma 3 now yields

$$
\begin{aligned}
& D_{12}<d_{12}, D_{13}<d_{13}, D_{23}<d_{23}, \\
& D_{14}>d_{14}, D_{24}>d_{24}, D_{34}>d_{34} .
\end{aligned}
$$

Note that at least two among $1^{\prime} 4^{\prime} 2^{\prime}, 2^{\prime} 4^{\prime} 3^{\prime}$, and $1^{\prime} 4^{\prime} 3^{\prime}$ must be obtuse. Suppose first that $1^{\prime} 4^{\prime} 2^{\prime}$ is obtuse. Since 142 is acute, and in light of (19) there holds

$$
\begin{aligned}
D_{14}^{2}-d_{14}^{2}+D_{24}^{2}-d_{24}^{2} & -2 D_{14} D_{24} \cos \left(1^{\prime} 4^{\prime} 2^{\prime}\right) \\
& +2 d_{14} d_{24} \cos (142)>0
\end{aligned}
$$

On the other hand, the cosine law shows that the expression on the left is $D_{12}^{2}-d_{12}^{2}$, which is negative by (19). This is a contradiction. Thus $1^{\prime} 4^{\prime} 2^{\prime}$ cannot be obtuse. Similarly, if $2^{\prime} 4^{\prime} 3^{\prime}$ were obtuse, there would hold holds

$$
\begin{aligned}
D_{24}^{2}-d_{24}^{2}+D_{34}^{2}-d_{34}^{2} & -2 D_{24} D_{34} \cos \left(2^{\prime} 4^{\prime} 3^{\prime}\right) \\
& +2 d_{24} d_{34} \cos (243)>0
\end{aligned}
$$

The left side is however $D_{23}^{2}-d_{23}^{2}$, which is negative by (19), again resulting in a contradiction. Thus $2^{\prime} 4^{\prime} 3^{\prime}$ cannot be obtuse, and a contradiction is obtained. 
Lemma 8. Suppose the triangle in Figure 6(a) represents the desired formation and the quadrilateral in Figure $6(d)$ represents an incorrect equilibrium shape. Suppose the angle 314 is acute. Then the formation on the right is unstable.

Proof: Suppose the incorrect quadrilateral in Figure 6(d) is stable. From Lemma 2, there must hold

$$
\begin{gathered}
D_{12}<d_{12}, D_{34}<d_{34} \\
D_{13}>d_{13}, D_{14}>d_{14}, D_{23}>d_{23}, D_{24}>d_{24}
\end{gathered}
$$

Suppose $z_{i}$ and $v_{i}$ are the positions for the agents of the triangle and quadrilateral formations, respectively. As the stability properties of the control law are rotation and translation invariant in both the desired and incorrect formation, we can without loss of generality assume that $z_{1}=v_{1}=0$. Then (20) implies

$$
\begin{aligned}
\left\|z_{2}\right\| & >\left\|v_{2}\right\| \\
\left\|z_{3}-z_{4}\right\| & >\left\|v_{3}-v_{4}\right\| \\
\left\|z_{3}\right\| & <\left\|v_{3}\right\| \\
\left\|z_{4}\right\| & <\left\|v_{4}\right\|
\end{aligned}
$$

Further, for the triangle formation for some $0<a_{i}<1$ there holds

$$
z_{2}=a_{3} z_{3}+a_{4} z_{4}
$$

On the other hand, for the quadrilateral formation for some $b_{i}>1$ there holds

$$
v_{2}=b_{3} v_{3}+b_{4} v_{4} .
$$

From (21), (25), and (26) we have

$$
\begin{gathered}
a_{3}^{2}\left\|z_{3}\right\|^{2}+a_{4}^{2}\|z 4\|^{2}+2 a_{3} a_{4} z_{3}^{T} z_{4}> \\
b_{3}^{2}\left\|v_{3}\right\|^{2}+b_{4}^{2}\|v 4\|^{2} \\
+2 b_{3} b_{4} v_{3}^{T} v_{4}
\end{gathered}
$$

Since angle 314 is acute, then

$$
z_{3}^{T} z_{4}>0 .
$$

Thus, from (23) and (24), and since $0<a_{i}<1$ and $b_{i}>1$, we have

$$
z_{3}^{T} z_{4}>v_{3}^{T} v_{4}
$$

From (22) there also holds

$$
\left\|z_{3}\right\|^{2}+\left\|z_{4}\right\|^{2}-2 z_{3}^{T} z_{4}>\left\|v_{3}\right\|^{2}+\left\|v_{4}\right\|^{2}-2 v_{3}^{T} v_{4}
$$

From (23) and (24) this implies

$$
z_{3}^{T} z_{4}<v_{3}^{T} v_{4}
$$

which establishes a contradiction.

Remark. Similar analysis is possible to show instability of all degenerate formations, in which three or more agents are collinear, or two or more agents are collocated. This analysis is omitted due to space limitations and will be included in a forthcoming journal version.

We have shown that with certain acuteness conditions on the desired formation, any possible incorrect equilibrium shape is unstable. In particular, if the desired formation is quadrilateral, any incorrect equilibrium shape is unstable if the diagonals make acute angles with the sides, and if the desired formation is triangular, any incorrect equilibrium shape is unstable if the angles of the large triangle are acute. It follows that the desired formation is almost globally asymptotically stable. While there may be initial conditions that converge to a saddle equilibrium, these belong to a set of measure zero; any perturbation results in convergence to the desired shape.

\section{CONCLUDING REMARKS}

This paper has considered a four-agent formation shape control problem using gradient-based interagent distance control laws with relative position measurements where the information architecture is a complete graph. We examined examples that feature equilibrium formation shapes with incorrect interagent distances and showed that these were unstable. More generally, we established that if the desired formation satisfies certain acuteness conditions, then any possible incorrect equilibrium shape is unstable. It follows from previous local stability results that despite the existence of possible incorrect equilibria, the desired formation is almost globally asymptotically stable.

Our preliminary study in this paper suggests several interesting further considerations. The first is an examination of whether or not the acuteness assumption is necessary, and if it is not then there is the challenge of removing it. Second, while we have shown that any unstable incorrect equilibrium shape is unstable, we have not counted how many there may be for a given desired shape. Morse theory may lead to a characterization (see e.g. [9]). Finally, one could determine whether and how there results might generalize to formations with more than four agents, and along these lines a particularly interesting question is what role is played by the information architecture properties, e.g. global rigidity. More precisely, one could ask whether similar results as those of this paper should be expected for a globally rigid 5 vertex graph, or might one need to consider the complete graph $K_{5}$ ?

\section{REFERENCES}

[1] L. Krick, M. Broucke, and B. Francis, "Stabilization of Infinitesimally Rigid Formations of Multi-Robot Networks," International Journal of Control, vol. 82, no. 3, pp. 423-439, 2009.

[2] T. Eren, P. Belhumeur, B. Anderson, and A. Morse, "A framework for maintaining formations based on rigidity," in Proceedings of the 15th IFAC World Congress, Barcelona, Spain, 2002, pp. 2752-2757.

[3] R. Olfati-Saber and R. Murray, "Distributed cooperative control of multiple vehicle formations using structural potential functions," in Proceedings of the 15th IFAC World Congress, Barcelona, Spain, 2002, pp. 346-352.

[4] 1 , "Graph rigidity and distributed formation stabilization of multivehicle systems," in Proceedings of the 41nd IEEE Conference on Decision and Control, Las Vegas, NV, 2002, pp. 2965-2971.

[5] J. Baillieul and A. Suri, "Information patterns and Hedging Brockett's theorem in controlling vehicle formations," 2003, pp. 556-563.

[6] J. Graver, B. Servatius, and H. Servatius, Combinatorial rigidity. American Mathematical Society, 1993.

[7] B. Jackson and T. Jordán, "Connected rigidity matroids and unique realizations of graphs," Journal of Combinatorial Theory, Series B, vol. 94, no. 1, pp. 1-29, 2005.

[8] R. Connelly, "Generic Global Rigidity," Discrete and Computational Geometry, vol. 33, no. 4, pp. 549-563, 2005.

[9] J. Milnor, Morse Theory. Princeton University Press, 1963. 